\title{
Rehabilitación en tiempos de COVID-19: panorama de algunas estrategias básicas
}

\author{
Rehabilitation in times of COVID-19: a panorama of some basic strategies
}

Dr. Emilio Frech López, ${ }^{*}$ Dra. Verónica Robles Saucedo*

* Instituto de Rehabilitación, Hospital Zambrano Hellion Tecnológico de Monterrey, Nuevo León, México.

Recibido: julio, 2020 Aceptado: julio, 2020
D esde finales del año 2019, se conocieron los primeros casos de infección por COVID-19 que es la enfermedad causada por el coronavirus que se ha descubierto más recientemente. Ya es calificada como una pandemia por la Organización Mundial de la Salud (OMS), y en la actualidad, México sufre consecuencias a gran escala, no sólo desde el punto de vista de salud de las personas, sino también afectaciones de tipo social, cultural, educacional, recreacional y económico, los sistemas que brindan servicios de salud, en particular de Medicina de Rehabilitación, no pueden abstraerse ni de los efectos propios de la enfermedad como tampoco de participar activamente en el combate a ésta.

Desde hace mucho tiempo, los servicios de rehabilitación participan en la atención de casos con síndrome de dificultad respiratoria aguda, desde su estancia en unidades de cuidados intensivos, durante el proceso de atención subagudo ${ }^{1-5}$, y después de su alta hospitalaria, contribuyendo de manera evidente a una mejora en la calidad de vida de las personas, sin olvidar beneficios en cuanto a menos días de hospitalización, prevención de complicaciones, diminución de recurrencias, menores costos de atención y una integración más pronta y de mayor calidad a las actividades cotidianas de los afectados.

De acuerdo con la OMS², el prestar servicios de rehabilitación es continuamente limitado u obstruido porque se califica de manera errónea como un servicio de salud no esencial, cuando para muchos casos sí es esencial. Los servicios que por lo general se limitan son tanto intrahospitalarios a los propios afectados por la COVID-19, como también a los pacientes que presentan en el hospital otras patologías, por ejemplo enfermedades cardiacas, accidentes cerebrovasculares, amputaciones, etcétera; por otro lado, los servicios posthospitalarios de rehabilitación también se limitan por este mismo factor, lo cual tiene como consecuencia el comprometer los resultados en cuanto a salud, y se incrementará en el futuro la necesidad de estancias más prolongadas en hospital así como readmisiones al hospital debidas a complicaciones.

Desde el inicio de la pandemia, han emergido múltiples publicaciones con el objetivo de generar guías para el manejo rehabilitador de estos casos.

En México, tenemos sistemas de salud que consideraríamos robustos, pero en otros casos con debilidades estructurales y de recursos humanos que facilitan desviaciones de la atención adecuada en el ámbito de la rehabilitación. No existen estadísticas en nuestro país que muestren la frecuencia de participación de servicios de rehabilitación en síndromes de dificultad respiratoria aguda, ni siquiera en enfermedades en general atendidas en Unidades de Cuidados Intensivos (UCI). Reportes de la OMS han mostrado que en el $63 \%$ de los países existen obstrucciones

Citar como: Frech LE, Robles SV. Rehabilitación en tiempos de COVID-19: panorama de algunas estrategias básicas. Rev Mex Med Fis Rehab. 2020; 32 (1-2): 25-29. https://dx.doi.org/10.35366/98516 
o limitaciones para prestar servicio de rehabilitación durante esta pandemia.

Parece importante, en el momento actual, generar guías basadas en la mejor evidencia disponible del cómo brindar servicios de rehabilitación de manera segura, tanto para el paciente como para el personal sanitario, costo efectivas, integrales, interdisciplinarias y con objetivos claros en tiempos y metas a lograr.

Es claro que el personal sanitario que participe tiene que tener las calificaciones adecuadas, debe incluir sin ninguna duda a médicos especialistas en Medicina de Rehabilitación, Intensivistas, Neumólogos, Infectólogos, Neurólogos, Internistas, etcétera, dependiendo de las características de cada paciente; no olvidar que el personal de salud asociado como los terapeutas físicos, ocupacionales, de lenguaje, ortesistas y protesistas, así como enfermería, nutrición y psicología entre otros cumplen un rol mayúsculo en la atención de estos casos.

Como en otros casos, será importante que cada persona del equipo de atención cumpla con su rol y sea respetuoso del de los demás, siempre basándose en las necesidades del paciente, este aspecto es de importancia máxima, en especial para equipos de reciente formación, o personal que no está acostumbrado a dinámicas de trabajo en equipo, debe resaltarse en particular el hecho de la participación de personal de Rehabilitación en Unidades de Cuidados Intensivos (UCI), donde se requerirá adaptarse a la dinámica de esta área sin obstruir su delicada actividad y ganar confianza tanto de los casos como del personal habitual ${ }^{4}$.

La participación deberá iniciar lo antes posible, siempre y cuando exista estabilidad hemodinámica (con o sin asistencia ventilatoria), para esta actividad deberá existir: 1) una solicitud de interconsulta a rehabilitación por el médico a cargo del paciente; 2) efectuar la interconsulta por parte de un médico especialista en Medicina de Rehabilitación; 3) una prescripción específica de actividades e intervenciones que llevarán a cabo terapeutas físicos y/u ocupacionales; 4) si se considera, se deberá incluir al especialista en Audiología, Otoneurología y Foniatría, en especial si el paciente requirió de asistencia ventilatoria o tuvo alteraciones en estado de conciencia; 5) disponibilidad de equipo de protección personal, adecuado a las características del paciente y del área física donde se vaya a intervenir, el personal deberá ser capacitado para la utilización de este equipo de protección personal; 6) reportes diarios de la evolución y respuesta a tratamientos prescritos por parte del Servicio de Rehabilitación con el objetivo de actualizar de manera dinámica las indicaciones; 7) siempre deberá estar desde las primeras etapas personal de inhalo terapia para manejo de secreciones de vías respiratorias. De acuerdo al consenso de Stanford Hall, se pueden enumerar las recomendaciones de rehabilitación en: a) generales; b) pulmonares; c) cardiacas; d) ejercicio; y e) psicológicas, las cuales deberán documentarse expresamente en las indicaciones para estos pacientes.

La OMS recomienda en la Tabla 1 una serie de actividades de acuerdo con la evolución del caso.

Algunos aspectos generales de la participación de personal de rehabilitación a tomar en cuenta son:

- En área de pacientes con pruebas de PCR aún positivas deberá usarse EPP especificado, en áreas donde los sujetos ya tienen PCR negativa, con protección habitual para sesiones de rehabilitación, que consiste en uso de cubrebocas y/o careta, ropa clínica que de preferencia deberá haberse vestido con ella al llegar a su lugar de trabajo y no traerla durante el traslado desde casa, lavado de manos antes y después de tocar al paciente, sanitizar el área al finalizar si es que están en área de uso común.

\section{INTERVENCIONES DE REHABILITACIÓN PARA PACIENTES CON COVID-19 SEVERA}

El rol que juegan los profesionales de rehabilitación en el manejo de pacientes con COVID-19 severo varía dependiendo del contexto, así como la manera en que las intervenciones son asignadas a diferentes disciplinas. Por ello, se debe interpretar e implementar esta guía basándose en la distribución de roles y el ámbito local. La Tabla 1 representa una compilación de evidencias relevantes de COVID-19 y rehabilitación, así como de aportaciones de expertos en rehabilitación respiratoria alrededor del mundo.

De acuerdo con los protocolos ya conocidos, las intervenciones de rehabilitación en pacientes en $\mathrm{UCI}$ pueden ser muy breves, desde 15 minutos hasta una hora, dependiendo el estado y tolerancia del paciente cada sesión y de las características individuales, pueden darse una o dos sesiones por día. Podríamos poner en orden de objetivos: 1) arco de movilidad; 2) manejo de secreciones incluyendo ejercicios para mecánica ventilatoria, drenaje postural y terapia de percusión; 3) fortalecimiento de miembros pélvicos y torácicos; 4) equilibrio de cuello; 5) equilibrio de tronco; 6) equilibrio de pie; y 7) marcha. Estos objetivos deberán llevarse de manera progresiva y secuencial teniendo la flexibilidad necesaria para poder en ocasiones intercalar su orden. Se sugiere monitorizar 
Tabla 1: COVID-19.

\begin{tabular}{|c|c|c|}
\hline Fase de cuidado & Intervenciones de rehabilitación & Lugar de intervención \\
\hline Agudo & $\begin{array}{l}\text { Los profesionales de rehabilitación pueden estar involucrados en el apoyo } \\
\text { de manejo respiratorio agudo de pacientes de COVID-19 recibiendo soporte } \\
\text { respiratorio para facilitar una temprana recuperación. Profesionales de } \\
\text { rehabilitación especializados pueden apoyar en mejorar la oxigenación, remover } \\
\text { secreciones de vías respiratorias y en el destete ventilatorio. Asimismo, estos } \\
\text { profesionales pueden promover la nutrición y prevenir neumonía por aspiración, } \\
\text { especialmente en casos que fueron intubados o tienen traqueotomía }\end{array}$ & $\begin{array}{l}\text { Unidad de Cuidados Intensivos } \\
\text { o Unidad de Alta Dependencia } \\
\text { (incluyendo centros de } \\
\text { tratamiento de infecciones } \\
\text { respiratorias severas agudas) }\end{array}$ \\
\hline Subagudo & $\begin{array}{l}\text { En el periodo temprano de recuperación, una vez que los pacientes hayan } \\
\text { egresado o nunca hayan entrado a una Unidad de Cuidados Intensivos o de } \\
\text { Alta Dependencia, las intervenciones de rehabilitación se pueden enfocar en } \\
\text { tratamientos de discapacidad de movilidad, función respiratoria, cognición, } \\
\text { comunicación, nutrición y ejercicios de deglución. Las intervenciones durante } \\
\text { este periodo buscan promover la independencia para actividades cotidianas } \\
\text { y proveer apoyo psicosocial. Los profesionales de rehabilitación también } \\
\text { contribuyen significativamente a los preparativos para el alta de los pacientes, lo } \\
\text { que es particularmente complejo con personas mayores o con comorbilidades }\end{array}$ & $\begin{array}{l}\text { Salas de hospital, Unidades } \\
\text { de Transición o Unidades } \\
\text { Subagudas (incluyendo centros } \\
\text { de tratamiento de infecciones } \\
\text { respiratorias severas agudas) }\end{array}$ \\
\hline Largo plazo & $\begin{array}{l}\text { Para los pacientes dados de alta, los profesionales de rehabilitación pueden } \\
\text { proveer ejercicios graduales, educación en conservación de energía, } \\
\text { modificación de hogar y comportamiento y productos asistenciales. Asimismo, } \\
\text { se puede proveer rehabilitación para discapacidades específicas individuales. } \\
\text { Durante la recuperación a largo plazo de COVID-19 severo, intervenciones de } \\
\text { rehabilitación pulmonar pueden ser de alto beneficio. Estas intervenciones se } \\
\text { enfocan en discapacidades físicas y respiratorias, e incluyen una combinación } \\
\text { de ejercicio gradual, educación, actividades cotidianas y apoyo psicosocial } \\
\text { En muchos contextos, las restricciones asociadas a la pandemia } \\
\text { (distanciamiento social, recursos humanos limitados y transporte público } \\
\text { reducido), así como los riesgos de infección, posiblemente implican el uso de } \\
\text { herramientas de telemedicina para pacientes dados de alta. Estas herramientas } \\
\text { se pueden usar para ejercicios remotos (como educación y ejercicio con "grupo } \\
\text { virtual») o para apoyo entre pacientes de COVID-19 que tengan entrenamiento } \\
\text { para dar dicho apoyo. Los servicios de rehabilitación en comunidades } \\
\text { normalmente están preparados para otorgar cuidados a largo plazo }\end{array}$ & $\begin{array}{l}\text { Centros de Rehabilitación, } \\
\text { Programas de Pacientes } \\
\text { Ambulatorios, Servicios en } \\
\text { Casa, Servicios Móviles, } \\
\text { Servicios de Telemedicina }\end{array}$ \\
\hline
\end{tabular}

OMS, OPS Consideraciones relativas a la rehabilitación durante el brote de COVID-19, Abr 28. 2020.

saturación de oxígeno, frecuencia cardiaca, tensión arterial y, en caso de estar disponible, EKG.

Con respecto al arco de movilidad, está descrito que realizar tres repeticiones de movilidad en todo el arco de movimiento dos veces al día es suficiente para mantener$\mathrm{lo}^{6}$, deberán siempre ser de manera gentil, en caso de restricciones realizar estiramientos suaves y sostenidos, en particular en aquellos segmentos en que son más comunes las limitaciones articulares como flexores de cadera, isquiotibiales, soleo/gemelos. En sujetos con afectaciones severas y que esperamos tiempos prolongados, aun en cama el uso de ortesis para mantener posición, prevenir contracturas y aliviar zonas de presión es fundamental.

Los ejercicios para mecánica ventilatoria podrán incluir, según el caso, fortalecimiento de músculos accesorios para la respiración, reeducación de mecánica respiratoria torácica y/o abdominal; de acuerdo con el neumólogo/intensivista, se verá la necesidad de oxígeno suplementario dependiendo el estadio de cada caso $^{7}$.

El trabajo de fuerza en $\mathrm{UCl}$ se hará de manera progresiva, con cargas y repeticiones bajas, se deberá trabajar por grupos musculares más que por vientres individuales $y$, si es posible, integrarlos a actividades ocupacionales, lo cual favorecerá un mayor interés del individuo y se alineará al beneficio funcional que se desea.

Por lo general, buscaremos seguir una secuencia de control de cuello, a control de tronco y a posición de pie, no parecería correcto intentar posición de pie si el caso no ha logrado un equilibrio en posición sedente ${ }^{8}$. El uso 
de grúas, camas de inclinación y equipos de suspensión, así como bastones y andadores podrán incorporarse a medida que el sujeto logre mejoras en fuerza. Las actividades de marcha al inicio se deberán hacer con asistencia y supervisión muy cercanas, es posible inclusive si el personal de la misma $\mathrm{UCI}$ permite hacerlo con asistencia ventilatoria en algunos casos.

Después de su egreso de la $\mathrm{UCl}$, en el periodo subagudo, como también se menciona en la Tabla 1, se buscará incrementar la independencia funcional del sujeto para actividades básicas cotidianas, la utilización de escalas funcionales que sean conocidas por el equipo de atención y sean factibles serán instrumentos de valor para ver los avances de los casos, así como ajustar medias terapéuticas para asegurar un alta en las mejores condiciones posibles; la atención, información y educación de cuidadores y familiares se deberá llevar a cabo de manera formal, ver las condiciones en las que el individuo vivirá al egresar y dar opciones de adaptaciones que pudieran facilitar la funcionalidad. La participación de personal de psicología, tanto para ayudar al paciente como a familiares, en algunos casos cobrará importancia. En esta etapa de planeación del alta, la atención y seguimiento de comorbilidades por el equipo de atención deberá asignarse de manera clara para no sobretratar al sujeto o crear confusión en indicaciones específicas de intervenciones tanto farmacológicas como no farmacológicas. De preferencia, al alta el sujeto deberá llevar indicaciones por escrito de estas intervenciones.

En el largo plazo, ya valoradas, en la presencia de limitaciones y dishabilidades que presenten los pacientes se instituirán medidas para revertirlas o minimizar sus efectos que deterioren la función y prevengan en lo posible la aparición de discapacidades.

Con respecto a la prescripción de ejercicio en este tiempo, se deberá hacer de manera precisa, es decir, si vamos a trabajar la parte de acondicionamiento aeróbico deberá ser claro el tipo, la intensidad y la duración de éste, así como los instrumentos para monitorear estas indicaciones, como son la frecuencia cardiaca o el índice de cansancio percibido (RPE), entre otros. Cuando se prescriba trabajo de fuerza, se deberán tener en cuenta las afectaciones específicas que se hayan generado durante la enfermedad o comorbilidades previas que se hayan o no exacerbado, ya que en particular la intensidad de estos trabajos puede tener repercusiones deletéreas si son demasiado altas o no generar el efecto deseado si son demasiado bajas, es poco probable que maniobras como sacar un número de repeticiones máximas (1RM o 10RM) sea adecuado porque la misma prueba sería peligrosa de realizar, así que en la mayoría de los casos se intentarán programas progresivos con monitoreo de respuesta a estas cargas, volvemos a los ejemplos del RPE, dinamometrías, pruebas de isocinesia, pruebas de marcha o salto, entre otros. Los ejercicios llamados de flexibilidad tienen el objetivo de mantener y/o mejorar los arcos de movilidad, lo que ayuda a una optimización y ahorro energético, así como prevenir otras complicaciones, deberán de realizarse de la manera que ya lo mencionamos; es en general aceptado que deban ser de manera suave y sostenida, para un adulto sano se considera que frecuencias de dos a tres veces por semana es suficiente, es probable que estos sujetos convalecientes se beneficien con frecuencias más altas. Dentro de estos trabajos aeróbicos de fuerza y flexibilidad, estarán invariablemente incluidas estrategias para mejorar mecánica ventilatoria.

El seguimiento de estos casos con las escalas funcionales, como lo mencionábamos en párrafos anteriores, deberá seguirse utilizando de manera continua y programada.

Como vemos, desde el punto de vista de la Medicina de Rehabilitación, el reto es grande en estos momentos y seguramente será mayor en el futuro cercano por la demanda de servicios de este tipo que se requerirán, y las limitaciones en estructura física y personal que existen no sólo en el país sino en el mundo, el estrés a nuestras estructuras de servicio deberá ser enfrentado con responsabilidad basada en evidencia, y que genere valor en la atención de nuestros casos y para la comunidad en general.

Este documento tiene como objetivo dar un panorama de algunas estrategias básicas para la rehabilitación, se recomienda adaptarse lo más cercano posible a estos lineamientos, de acuerdo a personal, espacio físico, recursos materiales y población que se atienda. Se recomienda la lectura de los documentos anexados en la bibliografía para un mejor entendimiento y aplicación específica de estas medidas sanitarias ${ }^{1,2,5,8,9}$.

\section{REFERENCIAS}

1. Thomas $\mathrm{P}$, Baldwin C, Boden I, Gosselink R, Granger CL, Hogson $\mathrm{C}$ et al. Physiotherapy management for COVID-19 in the acute hospital setting: clinical practice recommendations. J Physiother. 2020; 66: 73-82.

2. Organización Mundial de la Salud [sitio web]. Consideraciones relativas a la rehabilitación durante el bote de COVID-19. [Fecha de consulta: 28-08-2020]. Disponible en: https://iris.paho.org/ handle/10665.2/52104.

3. Hopkins RO, Spuhler VJ, Thonsen GE. Transforming ICU Culture to facilitate early mobility. Crit Care Clin. 2007; 23: 81-96.

4. Morris PE, Goad AR, Thompson CR, Taylor KM, Harry BM, Passmore LM et al. Early intensive care unit mobility therapy in the treatment of acute respiratory failure. Crit Care Med. 2008; 36 (8): 2238-2243. 
5. Barker-Davies R, O'Sullivan O, Pumi PK, Baker P, Cranley M, Dharm-Datta $S$ et al. The Stanford Hall consensus statement for post-COVID-19 rehabilitation. Br J Sports Med. 2020; 0: 1-11. doi:10.1136/bjsports-2020-102596.

6. Kottke FJ, Lehmann JF. Krusen's handbook of physical medicine and rehabilitation. 4a. ed. Philadelphia USA: Saunders, 1990.

7. Patel BK, Wolfe KS, Pohlman AS, Hall JB. Ease of mobilization during noninvasive ventilation in a randomized clinical trial of helmet ventilation in acute respiratory distress syndrome. Am J Respir Crit Care Med. 2017; 195: A2745.
8. Simosin R, Robinson L. Rehabilitation following critical illness in people with COVID-19 infection. Am J Phys Med Rehab. 2020; 99 (6): $470-474$.

9. Wade DT. Rehabilitation after COVID-19: an evidence-based approach. Clin Med (Lond). 2020; 20 (4): 359-365, 202007.

\author{
Correspondencia: \\ Dr. Emilio Frech López \\ E-mail: dr.emiliofrech@medicos.tecsalud.mx
}

